\title{
REVALIDAÇÃO DE CHORTINUS ALDRICH E CONSIDERAÇÕES SOBRE C. BEQUAERTI ALDRICH \\ COM DESCRIÇÃO DA TERMINÁLIA MASCULINA (DIPTERA, MUSCIDAE, CYRTONEURININAE)
}

\author{
Denise Pamplona ${ }^{1}$ \\ Márcia Souto Couri ${ }^{1,2}$
}

\begin{abstract}
Revalidation of Chortinus At.dRICH AND CONSIDFrations on $C$. BEQUAERTI ALDRICH WITH DESCRIPTION OF MALE TERMINALIA (DIPTERA, MUSCIDAE, CYrToneurininaE). Chortinus Aldrich, 1932 is a monobasic genus, with $C$. bequaerti Aldrich, 1832 as the only known described species, and was considered as a synonym of Cyrloneurina Giglio-Tos, 1893 by CARVAl.HO et al. (1993). Chortinus is herein revalidate, based on the examination of adults and original description. The male terminalia is herein described for the first time. A key to distinguish these two genera is also presented.

KEY WORDS. Diptera, Muscidae, Chortinus, taxonomy
\end{abstract}

ALDRICH (1932) descreveu Chortimus, designando como espécie-tipo Chortinus bequaerti, descrita de uma série de 4 machos e 19 fêmeas da Guatemala (Santa Emília, Pochuta). Após a descrição original, poucas referências foram feitas a este gênero, além de sua citação em catálogos.

ALBUQUERQUE \& LOPES (1983) apresentaram a redescrição do gênero e da espécie-tipo, com base no exame de dois parátipos machos e quatro fêmeas do "National Museum of Natural History" (Washington, D.C.). Descreveram ainda o ovo e a larva de terceiro ínstar e mencionaram a condição larvípara da espécie.

CARVALHO et al. (1993) propuseram a sinonímia deste gênero com Cyrtoneurina Giglio-Tos, 1893.

Com base no exame de exemplar comparado com o tipo, pertencente à coleção de Diptera do Museu Nacional, de um exemplar do "The Natural History Museum", Londres, na descrição original e na descrição de ALBUQUERQUE \& LOPES (1983), o gênero Chortinus está sendo revalidado. É apresentada a redescrição do gênero e, como a espécie-tipo está bem caracterizada na literatura, apresenta-se a redescrição da terminália feminina e descrição da masculina. Ao final das redescrições, são feitos comentários sobre os gêneros Chortinus e Cyrtoneurina.

1) Departamento de Entomologia, Museu Nacional, Universidade Federal do Rio de Janeiro. Quinta da Boa Vista, São Cristóvão, 20940-040 Rio de Janeiro, Rio de Janeiro, Brasil.

2) Bolsista do CNPq. 


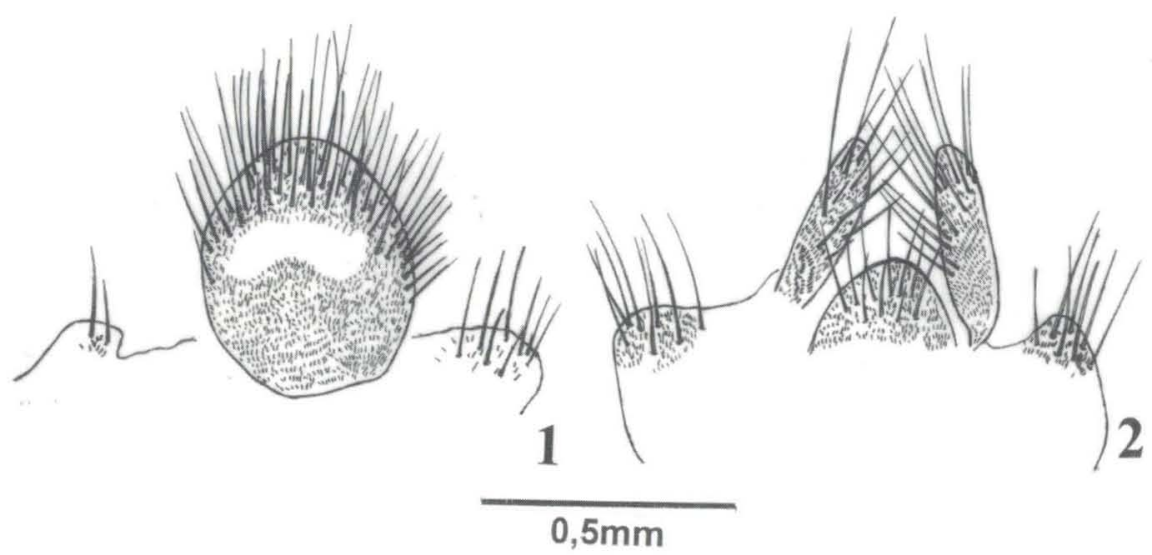

Figs 1-2. Chortinus bequaerti. (1) Ovipositor, vista dorsal; (2) ovipositor, vista ventral. Escalas $=0,2 \mathrm{~mm}$.

\section{Chortinus Aldrich, 1932}

Chortinus Aldrich, 1932: 23-25 (descrição). - Pont, 1972: 57 (catálogo). - Albuquerque \& Lopes, 1983:

191 (redescrição). - Couri \& Lopes (1985): 2 (chave).

Cyrtoneurina Giglio-Tos, 1893: 5-7. -Carvalho et al., 1993: 49 (catálogo, Syn.n.).

Espécie-tipo: Chortinus beaquaerti (des. orig.)

Diagnose. Comprimento total de 7,5-8,5mm. Olhos curta e esparsamente ciliados; machos com facetas ântero-internas maiores que as demais; machos holópticos e fêmeas dicópticas; arista curtamente ciliada em ambos os lados (comprimento dos cílios cerca da metade do comprimento do pedicelo); palpos pouco mais dilatados em direção ao ápice; cerdas acrosticais $0: 1$, cerdas dorso-centrais 2:4; intra-alares 0:2; pré-alar forte, semelhante às dorso-centrais; escutelo nu abaixo do nível das cerdas escutelares, na margem látero-ventral. Notopleura com duas cerdas longas. Prosterno nu, membrana em volta do prosterno nua; uma cerda proepisternal longa, voltada para cima, acompanhada de 4-6 menores; duas cerdas proepimerais voltadas para cima; propleura nua; anepisterno, catepisterno e anepímero com cílios de fundo longos; catepímero ciliado; espiráculo posterior bem maior que o anterior e sem cílios negros ou castanhos na borda inferior; caliptras nuas nas superfícies dorsal e ventral; caliptra inferior cerca do dobro do comprimento da superior; $R_{1}$ e $R_{4+5}$ ciliadas nas faces dorsal e ventral; veias $R_{4+5}$ e $M_{1+2}$ chegando ao ápice quase paralelas, ambas apresentando uma pequena ondulação um pouco antes de atingir o ápice; dm-cu sinuosa; remígio, na face dorsal, com cílios curtos no terço basal; coxa posterior, na face posterior nua; tíbia posterior na faces ântero-ventral, ântero-dorsal e póstero-dorsal com uma cerda curta no terço apical. Ovipositor curto; epiprocto largo, com microtríquias, com numerosas cerdas implantadas no terço apical; hipoprocto curto, com cerdas implantadas na metade apical, cercos mais longos que hipoprocto. Quinto esternito com angulação na superfície dorsal, na metade apical, placa cercal com reentrância lateral e parâmero maior que gonópodo e apódema do edeago bem esclerotinizado. 
Distribuição geográfica. Guatemala, Costa Rica e Panamá.

Considerações. Aldrich (1932) posicionou Chortinus próximo a Phaonia, diferindo deste principalmente pela ciliação nas veias da asa e presença ou ausência de cílios no anepímero.

\section{Chave para distinção de Chortinus de Cyrtoneurina}

1. Comprimento total do corpo: 7,5-8,5mm. Arista curtamente ciliada. Escutelo nu abaixo do nível das cerdas escutelares, na margem látero-ventral; cerdas intra-alares $0: 2 ; \mathrm{M}_{1+2}$ e $\mathrm{R}_{4+5}$ quase paralelas próximo ao ápice .........

Chortinus

- Comprimento total do corpo: 3,5-6,0 mm. Arista longamente ciliada. Escutelo com cílios abaixo do nível das cerdas escutelares, na margem látero-ventral; cerdas intralares $0: 1 ; \mathrm{M}_{1+2} \mathrm{com}$ uma discreta curvatura em direção à $\mathrm{M}_{1+2}$

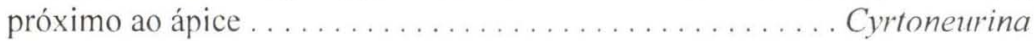

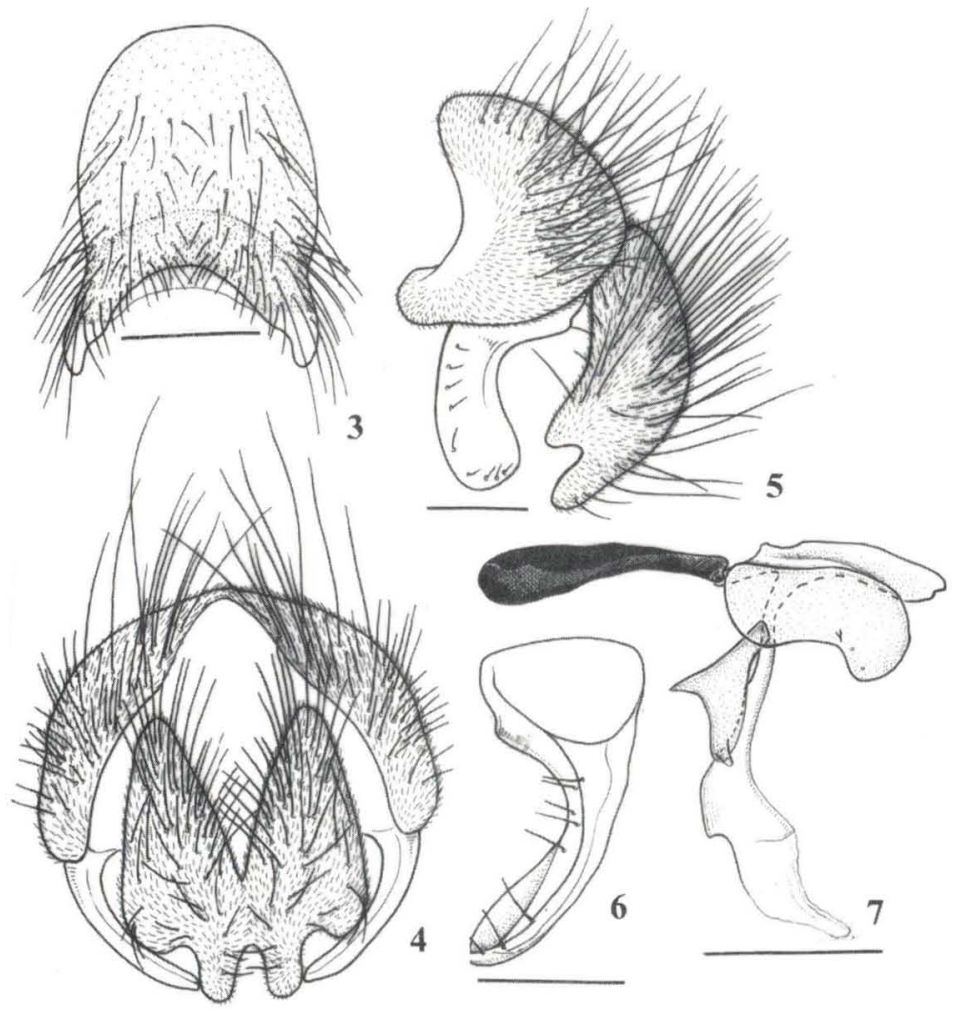

Figs 3-7. Chortinus bequaerti. (3) Quinto esternito, vista dorsal; (4) epândrio, placa cercal e surstilo, vista dorsal; (5) epândrio, placa cercal e surstilo, vista lateral; (6) surstilo direito, vista dorsal; (7) complexo fálico, vista lateral. Escalas $=0,2 \mathrm{~mm}$. 
Chortinus bequaerti Aldrich, 1932

Figs 1-7

Chortinus bequaerti Aldrich, 1932: 24-25 (descrição, macho e fềmea). - Pont (1972): 57 (catálogo), -Albuquerque \& Lopes, 1983: 191-193 (redescrição, macho e fềmea, descrição ovo e larva do terceiro instar e esqueleto céfalo-faringeano).

Caracterização da espécie. Ver ALdRICH (1932) e AlBUQUERQUE \& LOPES (1983), complementadas pela redescrição da terminália da fềmea e descrição da terminália do macho abaixo.

Fêmea. Terminália: ovipositor curto, não telescopado; epiprocto com muitas cerdas apicais (Fig. 1); hipoprocto com muitas cerdas na metade apical, sem concavidade central e reentrância; cercos cilíndricos e estreitos, visíveis apenas na face ventral, com cerdas longas, mais concentradas no ápice e na face interna (dobro do comprimento do hipoprocto) (Fig. 2).

Macho. Terminália: quinto esternito com leve angulação na superfície dorsal na metade apical (Fig. 3); ciliação no surstilo ausente, com cerdas na face interna e com ápice próximo à margem anterior da placa cercal; reentrância na margem anterior da placa cercal presente (Figs 4-6); parâmero maior que o gonóstilo, espinhos ausentes no edeago e cílios ausentes no gonópodo; apódema do edeago alargado apicalmente (Fig. 7).

Material examinado. Panamá, Cerro de Punta: Chiriqui, 1 fêmea, II-III1940. Costa RicA: San Jose: Copey (Dota), 1 macho, 19-VII-1974, L.F. Jirón leg. (NHM).

\section{REFERÊNCIAS BIBLIOGRÁFICAS}

Albuquerque, D. De O. \& S.M. Lopes. 1983. Redescrição de Chortinus bequaerti Aldrich, 1932 (Diptera, Muscidae, Cyrtoneurininae). Rev. Brasil. Biol. 43 (2): 191-194.

Aldrich, J.M. 1932. A new genus and two new species of muscoid flies from Guatemala. Proc. Ent. Soc. Wash. 34 (2): 23-26.

Carvalho, C.J.B.; M.S. Couri; A.C. Pont; D. Pamplona \& S.M. Lopes. 1993. Part II. Muscidae. In: C.J.B. CARVALHO (Ed.) A Catalogue of the Fanniidae and Muscidae (Diptera) of the neotropical region. São Paulo, Sociedade Brasileira de Entomologia, $201 \mathrm{p}$.

COURI, M.S \& S.M. LOPES. 1985. Neotropical genera of Coenosiinae -nomenclatural notes and key to identification (Diptera- Muscidae). Rev. Brasil. Biol. 45 (4): 589-595.

Pont, A.C. 1972. A Catalogue of the Diptera of the America South of the United

States. 97. Muscidae. São Paulo, Museu de Zoologia, Universidade de São Paulo, $111 \mathrm{p}$. 